\title{
FACTORES QUE INCIDEN EN EL DESEMPEÑO DE LA MIPYME DEL ESTADO DE QUERÉTARO
}

\section{FACTORS THAT AFFECT THE PERFORMANCE OF MSME (MIPYME) IN THE STATE OF QUERÉTARO}

\author{
Carmen Berenice Ynzunza Cortés*, Juan Manuel Izar Landeta**
}

* Doctora en Administración. Universidad Tecnológica de Querétaro. ORCID: https://orcid.org/0000-00031469-4763.

** Doctor en Administración. Instituto Tecnológico Superior de Rioverde. Email: jmizar@hotmail.com, ORCID: https://orcid.org/0000-0002-3187-6481.

Dirección para recibir correspondencia: bynzunza@uteq.edu.mx 
FACTORES QUE INCIDEN EN EL DESEMPEÑO DE LA MIPYME DEL ESTADO DE QUERÉTARO.

\section{RESUMEN}

OBJETIVO: Explorar los factores que inciden en el desempeño de la MIPYME del Estado de Querétaro.

MATERIAL Y MÉTODO: Es un estudio empírico de tipo cuantitativo, de la MIPYME del Estado de Querétaro de los sectores de servicios, comercio y manufactura, en el que participaron 707 empresas seleccionadas con base en el número de empleados, bajo un método de muestreo no probabilístico de conveniencia. La recolección de datos, se realizó mediante una encuesta aplicada a dueños y directivos, adaptada de Martínez et al., (2007) al contexto local. Para el análisis de datos, se utilizaron el análisis factorial exploratorio, de conglomerados jerárquico, así también técnicas estadísticas descriptivas e inferenciales.

RESULTADOS: Los hallazgos obtenidos, validan las relaciones existentes en el desempeño en función de la gestión estratégica, la tecnología y la innovación. La principal contribución, radica en la comprobación empírica de éstos como factores que afectan directamente el desempeño de la MIPYME.

CONCLUSIONES: Existe la necesidad de desarrollar estrategias y acciones para fortalecer los aspectos que limitan el desempeño y competitividad de la MIPYME.

PALABRAS CLAVE: Gestión estratégica. Tecnología. Innovación. Desempeño.

\section{ABSTRACT}

OBJECTIVE: To explore the factors that affect the performance of the MSME (MIPyME) in the State of Querétaro.

MATERIAL AND METHOD: It is an empirical study of a quantitative type, from the MSME (MIPyME) related to services, commerce and manufacturing sectors of the State of Querétaro, in which 707 companies that were chosen based on the number of employees participated under a non-probability sampling method of convenience. The data collection was carried out through a survey applied to owners and managers, adapted from Martínez et al., (2007) to the local context. 
FACTORES QUE INCIDEN EN EL DESEMPEÑO DE LA MIPYME DEL ESTADO DE QUERÉTARO.

For the data analysis, exploratory factorial analysis of hierarchical clusters, as well as descriptive and inferential statistical techniques were used.

RESULTS: The findings obtained validate the existing relationships in performance based on strategic management, technology, and innovation. The main contribution lies in the empirical verification of these as factors that directly affect the performance of the MSME (MIPyME).

CONCLUSIONS: There is a need to develop strategies and actions to strengthen the aspects that limit the performance and competitiveness of the MSME (MIPyME).

KEYWORDS: Strategic management. Technology. Innovation. Performance.

\section{INTRODUCCIÓN}

El papel de la Micro, Pequeña y Mediana Empresa (MiPyME) en el desarrollo económico y en la generación de empleos ha captado la atención de gobernantes y académicos de todo el mundo. En términos de empleo y número de empresas, la MiPyme domina las economías mundiales y produce beneficios importantes en la eficiencia económica, la productividad y la innovación. En México, tiene una contribución significativa a la economía y desarrollo del país, ya que conforma el $99.8 \%$ de las entidades productivas, genera el $52 \%$ del PIB y el $74.0 \%$ de los empleos totales (INEGI, 2014). Sin embargo, el nivel de competitividad alcanzado hasta ahora por la MiPyme mexicana, no le ha permitido crecer y posicionarse exitosamente en los mercados globales, debido a diversos factores internos y externos que obstaculizan y limitan su desarrollo. Entre ellos, se refieren sus características estructurales (Estrada, García y Sánchez, 2009), la falta de innovación en sus procesos y productos (Gardiner, 2015) y la gestión de sus recursos (Thi y Nico, 2015); además de los problemas relacionados con sus procesos y tecnología (Howard, 2014).

Querétaro, es uno de los estados del país que ha tenido una de las mayores tasas de crecimiento económico en los últimos años. De las 92,402 empresas que existen en el Estado, el 99.51\% son MiPyme; generan el $60.7 \%$ del empleo y aportan más del $50 \%$ de la producción total bruta. Del total de empresas instaladas, el $98.12 \%$ son micro y pequeñas empresas. El $43.2 \%$ pertenecen al sector comercio, $44.0 \%$ al de servicios, el $8.7 \%$ se dedican a la manufactura y un $4.1 \%$ engloba a otros sectores productivos minoritarios (DENUE, 2019). El sector manufactura, provee el YNZUNZA CORTÉS C. B., IZAR-LANDETA J. M. 
FACTORES QUE INCIDEN EN EL DESEMPEÑO DE LA MIPYME DEL ESTADO DE QUERÉTARO.

volumen más grande de empleos con $35.79 \%$, seguido del de servicios con $31.7 \%$ y $25.0 \%$ del sector comercio. El primero, es el más representativo del Estado, ya que proporciona más del $70.2 \%$ de la producción bruta total; la cual es generada en gran medida por empresas grandes, muchas de ellas trasnacionales, en contraste con la participación de la MiPyme local que es todavía muy baja (INEGI, 2014).

En el Estado, están presentes todas las condiciones para el surgimiento y consolidación de estas empresas, sin embargo prevalece aún la apertura de micro y pequeñas unidades de negocio, sobre todo del sector servicios, contrario a empresas de manufactura, las cuales han mostrado la menor tasa de crecimiento anual en los últimos años (INEGI, 2014; DENUE, 2019). Por lo que, la identificación de áreas de oportunidad, podría contribuir al establecimiento de acciones para su crecimiento y consolidación. Aunado a ello, son pocos los estudios en el país, sobre la competitividad y el número de PyMEs no competitivas. De igual forma, la evidencia empírica sobre los aspectos internos y estructurales de la MIPyME, asociados a la gestión como son: la planeación estratégica, la estructura organizacional y el establecimiento de alianzas estratégicas; así también con la tecnología e innovación, es aún limitada y éstos han sido abordados de forma aislada. En virtud de ello, el objetivo de investigación planteado, busca determinar los factores que inciden en el desempeño de la MIPyME del Estado de Querétaro. Para lo cual, se hace

primeramente la revisión de literatura relacionada y el planteamiento metodológico, y en seguida se procede al análisis y discusión de los resultados, así como a la presentación de las conclusiones, limitaciones y recomendaciones.

\section{Gestión estratégica y desempeño}

Las empresas más allá de su tamaño, requieren del establecimiento de estrategias para enfrentar los cambios tecnológicos, competitivos y alcanzar una ventaja competitiva (Masocha, 2014). La teoría de los recursos y capacidades organizacionales, enfatiza la identificación de las actividades clave y la creación de recursos y capacidades estratégicas para mantener una posición competitiva (Barney, 1991; Grant, 1996). De igual manera, la teoría del capital intelectual, refiere a los activos intangibles: gente, relaciones, sistemas y procesos (capital humano, relacional y organizacional) en la creación de valor, innovación y ventaja competitiva (Edvinsson y Malone, 1999). De ahí, que cada día surjan más señalamientos sobre la importancia de fortalecer el desarrollo de los procesos nucleares o estratégicos de las organizaciones (Savaneviciene, Rutelione y Ciutiene, 2014). 
FACTORES QUE INCIDEN EN EL DESEMPEÑO DE LA MIPYME DEL ESTADO DE QUERÉTARO.

En este marco de gestión estratégica, se menciona por supuesto a la planeación, como el paso esencial en la formulación de las estrategias de negocio que permiten dirigir el rumbo y los esfuerzos de la empresa para anticipar o responder de forma oportuna a las condiciones variantes del entorno (Boyne y Walker, 2004; Leyva, Cavazos y Espejel, 2018; Cokins, 2017). Igual, asume un papel preponderante en la asignación y aplicación efectiva de recursos (Glaister et al, 2008) y al estar vinculada a otras capacidades que agregan valor a la organización, puede afectar el desempeño operativo, la rentabilidad y el rendimiento organizacional. Así también, la supervivencia y el crecimiento, especialmente tratándose de empresas pequeñas (Gruber, 2007; Sosiawani et al, 2015; Gica y Negrusa, 2011). No obstante, se halla que en muchos casos, el proceso de planeación estratégica no está bien estructurado; es más bien esporádico e informal y no se visualiza como un elemento clave para hacer frente a escenarios futuros adversos y/o capitalizar oportunidades (Kraus, Harms y Schwarz, 2006).

De igual forma, se señala la importancia de la estructura organizacional en la implementación de la estrategia y su alineación con las circunstancias del contexto interno y externo, dados los beneficios que la misma tiene en el proceder e interacción de los individuos y las organizaciones (Kanten, Kante y Gurlekc, 2015). La creación de la estructura organizacional, representa no solo el esquema de configuración de las actividades (Amitabh y Gupta, 2010), sino también los aspectos de centralización y formalización de las mismas, los cuales tienen una incidencia significativa en el desempeño organizacional (Sahay y Gupta, 2011); Liao, HuiChuang, Laitoc, 2011; Chan y Seok, 2014; Lee y Yang, 2011). Es también, el medio a través del cual las organizaciones se integran y diferencian para el logro de los objetivos empresariales y el desarrollo de innovación (Tran y Tian, 2013). En empresas pequeñas, puede ser una estructura simple, no estructurada y centralizada, con un bajo grado de formalización y no necesariamente un método para mejorar el desempeño (Nicolescu y Niscolescu, 2009).

Bajo esta misma visión, están las alianzas como una solución tangible a diversas limitantes que enfrenta la MiPyme; una alternativa estratégica y organizacional para robustecer las capacidades organizacionales; los aspectos tecnológicos y un mecanismo para acceder a otros recursos y oportunidades de negocio, a través del desarrollo de nuevos productos y/o la incursión en otros nichos o mercados (Mazzarol et al., 2014; Minarelli, Raggi y Viaggi, 2013) que resulten en un mejor desempeño y un crecimiento sostenido (Street y Cameron, 2007; Brouthers, Nakos y Dimitratos, 2015; Gronum, Verreyne y Kastelle, 2012). En particular, si estas alianzas, se gestan en torno a las grandes empresas con fines de abastecimiento de la cadena de proveeduría y con 
FACTORES QUE INCIDEN EN EL DESEMPEÑO DE LA MIPYME DEL ESTADO DE QUERÉTARO. aquellas que poseen mayores capacidades tecnológicas. Con base en este sustento teórico, se formula la siguiente hipótesis de investigación:

H1: A mayor gestión estratégica (planeación estratégica, estructura organizacional y establecimiento de alianzas estratégicas), mayor desempeño.

\section{Tecnología y desempeño}

La tecnología, es otro de los recursos asociados al desempeño organizacional y la ventaja competitiva (Barney, 1991; Grant, 1996). La inversión en nuevas tecnologías, para la fabricación o la gestión, mejora no solo la eficiencia operativa y la capacidad instalada, lo cual conlleva a un mejor desempeño (Kotha, 1991; Bülbül et al., 2013), sino también agrega valor a las organizaciones (Aral y Weill, 2007), ya que permite a las empresas alcanzar una mayor madurez tecnológica (Mittal, Romero y Wuest, 2018), con lo cual flexibiliza la manufactura y propicia la reconfiguración de los procesos, lo que facilita el diversificar la producción; incrementar los volúmenes y por ende las utilidades, asegurando así, una mayor competitividad y la permanencia en el mercado de las organizaciones (Edquist, 2001; Heidenreich, 2009; Kotha, 1991). Por otro lado, las tecnologías de la información, hacen posible el mejoramiento de los procesos productivos; contribuyen a facilitar la gestión y al fortalecimiento de las capacidades organizacionales y gerenciales (Aral y Weill, 2007; Jeffers, Muhanna y Naul, 2008), lo cual incide de forma directa o indirecta en la rentabilidad, la competitividad y el crecimiento de las organizaciones (Ghobakhloo et al., 2011; Mithas et al., 2012). Asimismo, favorecen el acceso a fuentes externas de conocimiento, de innovación y ventaja competitiva (Heidenreich, 2009; Mithas et al., 2012; Hervas, Sempere y Boranat, 2014). Esta argumentación teórica sustenta la hipótesis de investigación siguiente:

H2: A mayor tecnología (para los procesos y la gestión), mayor desempeño.

\section{Innovación y desempeño}

La innovación, desde la perspectiva estratégica de cualquier organización, es igualmente un recurso organizacional crítico para alcanzar un mejor desempeño y factor clave para que las empresas sobrevivan y generen una ventaja competitiva (Jiménez y Sanz, 2011), principalmente en el caso de pequeñas empresas (Shapiro et al., 2015). Como resultado de la innovación, las organizaciones pueden ampliar la oferta de sus productos y servicios o crear nuevos mercados (Aamir, Satirenjit y Mohammad, 2017; Aminreza, Maryam y Lagha, 2011). Estas innovaciones, YNZUNZA CORTÉS C. B., IZAR-LANDETA J. M. 
FACTORES QUE INCIDEN EN EL DESEMPEÑO DE LA MIPYME DEL ESTADO DE QUERÉTARO.

pueden estar relacionadas con los procesos, la gestión o la generación de nuevos productos (OECD, 2009) a fin de mejorar la productividad o competitividad, especialmente en aquellas empresas dedicadas a la manufactura o los servicios (Mairesse, Mairesse y Robin, 2009; Ndemezo y Kayitana, 2017). De ahí, que para alcanzar un desempeño superior, se sugiera que las empresas transiten de la innovación tecnológica y de gestión, que solo impactan la efectividad organizacional; la estructura y el sistema operativo de la organización (Damanpour, 2009) hacia la innovación de productos, dada la tendencia de las organizaciones a innovar mayormente en los dos primeros tipos de innovación (Sharma, 2014; Laforet y Tann, 2006). A partir de lo anteriormente expuesto, se plantea el supuesto siguiente:

H3: A mayor innovación (productos, procesos y gestión), mayor desempeño.

\section{Gestión estratégica, Tecnología, Innovación y Desempeño}

Investigaciones previas, vinculadas a la gestión estratégica han encontrado que las pequeñas empresas tratan a través del establecimiento de alianzas y estrategias para optimizar sus procesos y realizar pequeñas mejoras a sus productos (Van de Vrande et al., 2009). De igual forma, buscan adecuar su estructura organizacional, ya que ésta afecta el desarrollo de la innovación, el desempeño y la adaptación de las organizaciones (Sahay y Gupta, 2011; Liao et al., 2011; Chan y Seok, 2014; Lee y Yang, 2011). De ahí, que tanto para el establecimiento de alianzas como para el desarrollo de innovación, se requiera de un trabajo de planeación y preparación de largo plazo que asegure el éxito de las mismas (Dyer, Gregersen y Christensen, 2011; Hoffman y Schosser, 2001).

Por otra parte, se señala que tanto la innovación como el desempeño se ven afectados por la tecnología (Ndemezo y Kayitana, 2017), debido a que la incorporación de ésta involucra cambios en los métodos y procesos de trabajo, al igual que resulta en una reorganización de la producción, mejoras de ingeniería y propuestas de desarrollo de nuevos productos y servicios (Kennedy, 2006). Al mismo tiempo que, fortalece la estructura del negocio (Kaplan y Norton, 2008). Bajo esta argumentación teórica se plantea la hipótesis siguiente:

H4: A mayor gestión estratégica, tecnología e innovación, mayor desempeño organizacional. 
FACTORES QUE INCIDEN EN EL DESEMPEÑO DE LA MIPYME DEL ESTADO DE QUERÉTARO.

\section{MATERIAL Y MÉTODO}

El presente trabajo, presenta un estudio empírico realizado bajo un enfoque cuantitativo de tipo descriptivo y correlacional, cuya unidad de análisis fue la MiPyME de los sectores de manufactura, servicios y comercio de la zona metropolitana del Estado de Querétaro, registradas en el Directorio SIEM. La elección de la muestra, se hizo considerando el número de empleados, referente válido para clasificar el tamaño de las organizaciones como microempresas de 2 a 10 trabajadores, pequeñas de 11 a 50 y medianas de 51 a 250 empleados. Para lo cual, se utilizó un método de muestreo no probabilístico de conveniencia, con base en el deseo de los encuestados de participar en el estudio. El total de empresas participantes fue de 707; de éstas 216 fueron micro empresas, 327 pequeñas y 164 medianas. La obtención de la información, se llevó a cabo a través de la aplicación de una encuesta en persona al director, dueño o gerente de la empresa. Para el diseño del instrumento de medición, se hizo uso de la escala desarrollada por Martínez., et al. (2007) adaptada al contexto local. La encuesta consistió de 35 preguntas estructuradas, las cuales exploran aspectos generales y relacionados con la gestión, tecnología, innovación y el desempeño.

Como variables independientes se consideraron: 1) la planeación estratégica. En este caso, se solicitó a los participantes que indicarán si realizaban planeación estratégica dentro de sus organizaciones y el plazo de la misma. 2) si llevaban a cabo alianzas o acuerdos de colaboración y para qué fines: comercializar sus productos, compras y abastecimientos, logística, producción y/o investigación; $y, 3$ ) si tenían una estructura organizacional formal, con departamento de ventas, recursos humanos, investigación y desarrollo, contabilidad, calidad y producción.

En lo que concierne a la tecnología, se abordaron los aspectos vinculados con la tecnología para la fabricación y de la información (infraestructura y sistemas) para el soporte de la operación y gestión. Respecto de la primera, se les pidió a los participantes que indicaran el nivel de tecnología que poseía la empresa para llevar a cabo sus procesos de fabricación (débil, sostenible, buena y fuerte). Igual, se hizo respecto de las tecnologías de la información, considerando el uso del intranet, página web, mercadotecnia y comercio electrónico, así también el manejo de sistemas de información (gerencial, de control, auditoría, calidad y costos). En referencia a la innovación, se les preguntó a los empresarios si habían realizado innovación de productos, procesos y gestión en los últimos 3 años y la importancia de la misma. Con base en las respuestas, a los ítems de cada uno de los constructos, se estimaron las medias y se construyeron las variables respectivas.

YNZUNZA CORTÉS C. B., IZAR-LANDETA J. M.

MAYO-AGOSTO 2020. Año 26, Número 75. Págs. 139-156 
FACTORES QUE INCIDEN EN EL DESEMPEÑO DE LA MIPYME DEL ESTADO DE QUERÉTARO.

El desempeño, se estableció como la variable dependiente. Para medir el mismo, se incluyeron ítems asociados con cuestiones contables, financieras y de rendimiento en comparación con los principales competidores, utilizando 8 indicadores de acuerdo a la aproximación propuesta por Quinn y Rohrbaugh (1983) buscando a través de ellos, estimar un indicador global de éxito que permitiera agrupar a las empresas como aquellas con mejor y peor desempeño. Paralelamente, se emplearon el tamaño y edad de las organizaciones como variables de control. La primera, se estableció en función del promedio de empleados y la segunda, a partir de los años transcurridos desde la constitución o inicio de actividades de la empresa.

Con respecto a la operatividad y validez del instrumento de medición, se utilizó el Alfa de Cronbach, el valor alcanzado fue 0.845. Los estimados por variable, estuvieron entre el rango de 0.808 a 0.869 . Para validar las hipótesis, que se formularon en la investigación y comprobar la relación entre los factores, se utilizó el modelo de regresión lineal por Mínimos Cuadrados Ordinarios (MCO). Esto en virtud, de su adecuación a la naturaleza de las variables objeto de estudio. El modelo de investigación general propuesto, fue: Desempeño $=\beta o+\beta_{1} X_{1}$ Gestión estratégica (planeación estratégica, estructura organizacional, alianzas estratégicas) $+\beta_{2} X_{2}$ Tecnología (información, proceso) $+\beta_{3} X_{3}$ Innovación (producto, proceso, gestión) $+\beta_{4} X_{4}$ Tamaño $+\beta_{5} X_{5}$ Edad $+\varepsilon_{6}$

En cuanto a las características de la unidad muestral, están incluidas empresas de los tres sectores económicos, servicios, manufactura y comercio de tamaño micro, pequeño y mediano. El promedio de edad de las empresas, es 12.5 años y el de empleados de 23 años. Entre algunas de sus particularidades, está el que son preferentemente pequeñas empresas, constituidas pocas como sociedades y en su gran mayoría son de tipo familiar (ver tabla 1).

Tabla 1

Características de la unidad muestral

\begin{tabular}{lllllll}
\hline Sector & Manufactura & $37.0 \%$ & Comercio & $29.3 \%$ & Servicios & $33.7 \%$ \\
Tamaño & Micro & $30.6 \%$ & Pequeña & $46.3 \%$ & Mediana & $23.1 \%$ \\
Edad & $17.1 \%$ & $42.8 \%$ & $40.1 \%$ & & & \\
(años) & $0-3$ & $4-10$ & $>10$ & & & \\
Régimen & Pequeños & $14.3 \%$ & Persona & $38.2 \%$ & Persona & $47.5 \%$ \\
fiscal & contribuyentes & & Física & & Moral & \\
Tipo de & Familiar & $60.7 \%$ & Sociedad & $39.3 \%$ & & \\
empresa & & & & & & \\
\hline
\end{tabular}

Fuente: Elaboración propia.

YNZUNZA CORTÉS C. B., IZAR-LANDETA J. M.

MAYO-AGOSTO 2020. Año 26, Número 75. Págs. 139-156 


\section{RESULTADOS}

Para determinar las dimensiones propuestas en el modelo y medir el desempeño de la MiPyME, se utilizó el análisis factorial exploratorio con el método de componentes principales y rotación Varimax. El análisis factorial, es una técnica estadística multivariante que facilita la identificación de la estructura de los datos (Hair et al, 1999); es decir, grupos de variables homogéneas altamente correlacionadas. Los resultados de la medida Kaiser-Meyer-Olkin de adecuación de la muestra, fueron de 0.721 y la prueba de esfericidad de Bartlett de 1541.875 con 28 grados de libertad. Los valores de las comunalidades de las variables exploradas, estuvieron entre el rango de 0.595 y 0.915 . La tabla 2 , muestra la matriz de componentes rotados, en la cual se observa que los pesos de las cargas factoriales están por encima de 0.5 , lo que es un indicador válido para aceptar que un elemento sea parte de un factor (Hair et al., 1999). La reducción de componentes, resultó en tres factores que explican el $66.61 \%$ de la varianza total. El de mayor peso, está relacionado con la tecnología (22.96\%), seguido de la gestión estratégica $(22.21 \%)$ y la innovación (21.44\%).

Tabla 2

Matriz de componentes rotados

\begin{tabular}{cccc}
\hline \multirow{2}{*}{ Variables } & \multicolumn{3}{c}{ Componente } \\
\cline { 2 - 4 } & Tecnología & Gestión estratégica & Innovación \\
\hline INNOVGEST & .079 & .049 & .851 \\
INNOVPROD & -.096 & .142 & .741 \\
INNOVPROC & .110 & .242 & .611 \\
TECPROCES & .939 & .140 & .081 \\
TECINFORM & .956 & .001 & -.006 \\
ESTORGAN & .049 & .805 & .156 \\
ALIANESTRA & -.006 & .715 & .177 \\
PLANESTRA & .104 & .719 & .080 \\
$\%$ Varianza explicada & 22.963 & 22.215 & 21.441 \\
\% Varianza acumulada & 22.963 & 45.178 & 66.619
\end{tabular}

Método de extracción: análisis de componentes principales.

Fuente: Elaboración propia.

Con el fin de tener una primera aproximación a los factores así identificados, las empresas se clasificaron en dos grupos de acuerdo a su nivel de desempeño: alto y bajo. Para lo cual, se utilizó el análisis de conglomerados jerárquico, empleando el desempeño como la variable de 
FACTORES QUE INCIDEN EN EL DESEMPEÑO DE LA MIPYME DEL ESTADO DE QUERÉTARO.

agrupamiento. Esta técnica, permite agrupar a las empresas de acuerdo al grado de homogeneidad interna y heterogeneidad externa respecto de una o más variables. También, conocer las características de similitud, importancia y significación estadística de los grupos (Stock y Zacarías, 2011). Los resultados, muestran que de las 707 empresas, 432 tienen un bajo desempeño, lo que representa el $61.1 \%$ de las empresas participantes y 275 de éstas, refieren un desempeño alto (38.9\%). De acuerdo a estos hallazgos, las empresas con mejor desempeño se caracterizan por presentar valores medios superiores a la media total en todas las variables exploradas.

Para validar el efecto supuesto, de los factores determinantes del desempeño organizacional en la MiPyME, se procedió a comprobar si había diferencias entre los tres factores hallados en la estructura factorial y el desempeño organizacional. La tabla 3, muestra que las diferencias son significativas para los tres factores; es decir, hay cambios en el desempeño en función de estas variables. Las puntuaciones medias más altas, fueron para la gestión estratégica (1.7185), innovación (1.5534) y tecnología (1.5461) en ese orden, todas en empresas con desempeño alto. También, se verificó que existiera una relación lineal simple de cada variable independiente con la variable dependiente. Los resultados fueron positivos y significativos para la gestión estratégica $(\beta=0.206, p=0.000)$, la tecnología $(\beta=0.249, p=0.000)$ y la innovación $(\beta=0.251, p=0.000)$ con respecto del desempeño, probando así las hipótesis $\mathrm{H} 1, \mathrm{H} 2$ y H3 planteadas. El Valor Máximo de Inflación de Varianza (VIF) encontrado, permite descartar problemas de colinealidad entre variables.

Tabla 3

Resultados del análisis de varianza y regresión simple de los factores

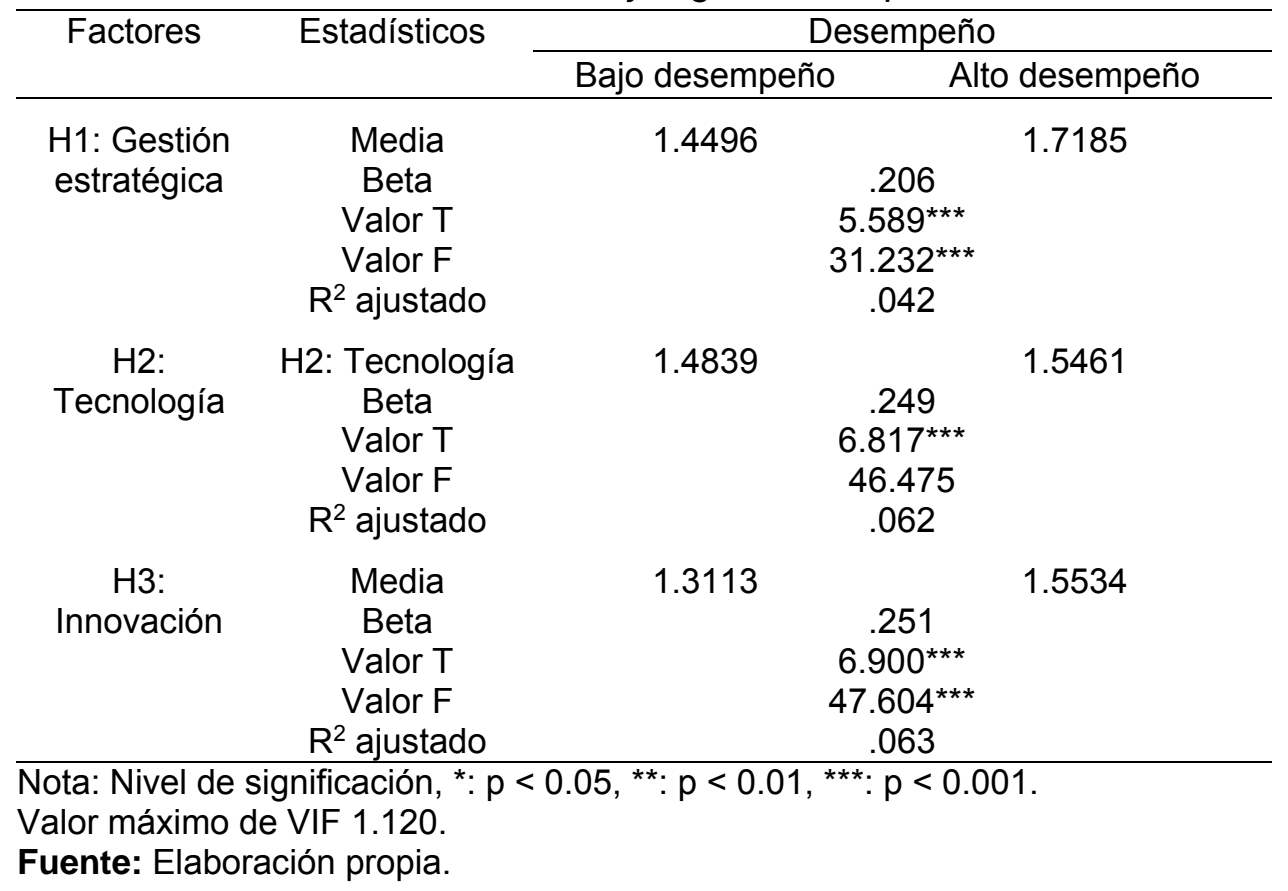

YNZUNZA CORTÉS C. B., IZAR-LANDETA J. M. 
FACTORES QUE INCIDEN EN EL DESEMPEÑO DE LA MIPYME DEL ESTADO DE QUERÉTARO.

Finalmente, para contrastar la hipótesis $\mathrm{H} 4$ y validar el modelo general propuesto, bajo el supuesto de que los tres factores determinados no son excluyentes, se integraron simultáneamente al modelo de regresión múltiple junto con las variables de control edad y tamaño, por considerar que éstas últimas también tienen un efecto importante en el desempeño. En la tabla 4, se presentan los resultados del modelo, el cual es significativo de acuerdo a los valores de F de 17.147 ( $p=0.000$ ) hallados; sin embargo, el poder de predicción es bajo, ya que se obtuvo un $\mathrm{R}^{2}$ ajustado de 0.103 , no obstante se comprueba que los tres factores conjuntamente tienen un mayor poder de predicción sobre el desempeño; al comparar las betas, se observa que el mayor peso corresponde a la innovación $(\beta=0.187, p=0.000)$, seguido de la tecnología $(\beta=0.173, p=0.000)$ y la gestión estratégica $(\beta=0.083, p=0.05)$. Los valores son positivos y significativos para cada uno de ellos. También, se prueba que la edad tiene un efecto significativo en el desempeño de las organizaciones $(\beta .084, p=0.025)$. No así el tamaño, dado que el resultado de la regresión fue no significativo y negativo $(\beta .-010, p=0.253)$.

Tabla 4

Análisis de la regresión del Modelo: Gestión estratégica, tecnología, innovación y desempeño

\begin{tabular}{lc}
\hline Variable & Desempeño \\
\hline Gestión estratégica & $.083^{*}$ \\
Tecnología & $.173^{\star * *}$ \\
& $(3.194)$ \\
Innovación & $.187^{* * *}$ \\
& $(4.910)$ \\
Tamaño & -.010 \\
& $(-.253)$ \\
Edad & $.084^{*}$ \\
& $(2.252)$ \\
VIF más alto & 1.422 \\
F/Sig. & $17.147^{* * *}$ \\
$\mathrm{R}^{2}$ ajustada & $(.103)$ \\
\hline Nota: Nivel de significación, ${ }^{*}: p<0.05,{ }^{* *}: p<0.01,{ }^{* * *}: p<0.001$. \\
Valor Máximo de Inflación de Varianza (VIF) 1.047. \\
Fuente: Elaboración propia.
\end{tabular}

\section{DISCUSIÓN Y CONCLUSIONES}

Este estudio empírico, busca contribuir a la determinación de los factores que inciden en el desempeño y la competitividad de la MiPyME. Con relación a los hallazgos, se prueba que la gestión estratégica, la tecnología y la innovación afectan el desempeño de las organizaciones. YNZUNZA CORTÉS C. B., IZAR-LANDETA J. M. 
FACTORES QUE INCIDEN EN EL DESEMPEÑO DE LA MIPYME DEL ESTADO DE QUERÉTARO.

También, que las empresas que presentan los valores más altos en los factores explorados son las que refieren los mejores indicadores. En virtud de estos resultados, se puede afirmar que a mayores niveles de cada uno de ellos es posible esperar un desempeño superior en comparación con los competidores principales.

Estos hallazgos, son similares a los obtenidos en trabajos previos, en los cuales se ha probado también la importancia de la gestión estratégica relacionada con la planeación estratégica, la estructura organizacional y el establecimiento de alianzas y/o acuerdos de colaboración con el desempeño, la innovación y el crecimiento de las organizaciones (Glaister et al., 2008; Gruber, 2007; Damanpour, 2009; Chan y Seok, 2014; Mazzarol et al., 2014; Street y Cameron, 2007; Brouthers et al., 2015). Bajo estos términos, la MiPyME podrá mejorar sus resultados organizacionales y alcanzar una ventaja competitiva, siempre que su gestión estratégica sea fortalecida, buscando a través de ella reducir sus deficiencias estructurales, acceder a recursos e incrementar sus capacidades.

De igual forma, es manifiesta la importancia de la tecnología para el desempeño organizacional, dado que se encontró que las empresas que poseen una tecnología fuerte, son igualmente las que parecen haber tenido un mejor desempeño; sin embargo, a la luz de estudios previos, los resultados no pueden considerarse como concluyentes, debido que existen diferencias en el sentido de las interrelaciones. No obstante, hay similitudes en cuanto a que tanto la tecnología para fabricación como de la información, tienen un efecto indirecto en el desarrollo de otros procesos organizacionales nucleares relacionados con la manufactura, la gestión y la innovación, los cuales han sido vinculados directamente al desempeño (Edquist, 2001; Heidenreich, 2009; Hervas et al., 2014; Mithas et al., 2012). Bajo este contexto, la MiPyME deberá visualizar las ventajas de incorporar tecnología, no solo para mejorar la gestión y eficiencia operativa, sino también para acceder a nuevo conocimiento y generar innovación. A razón de ello, la inversión en tecnología deberá ser considerada dentro del plan estratégico como una actividad prioritaria de mediano plazo a fin de obtener mejores logros organizacionales y mantenerse competitiva.

En cuanto a la innovación, los hallazgos son similares a los de trabajos precedentes, en los cuales se ha demostrado el vínculo existente entre el desempeño, la innovación y la ventaja competitiva (Sharma, 2014; Laforet y Tann, 2006). En este sentido, es clara la posición que deberá asumir la MiPyME respecto de la innovación, dado que el tamaño no debiera ser una limitante para innovar, ni una actividad exclusiva de empresas grandes. Para lo cual, es necesario que la MiPyME 
FACTORES QUE INCIDEN EN EL DESEMPEÑO DE LA MIPYME DEL ESTADO DE QUERÉTARO.

reconozca y valore algunas de sus capacidades como su flexibilidad operativa, de transformación e integración y su estructura no centralizada, si quiere mejorar su desempeño y crecimiento. Específicamente, las asociadas con la gestión estratégica, puesto que de ésta depende el acceso a otros recursos y capacidades, al igual que mayores oportunidades de mercado.

De acuerdo a los resultados alcanzados, se concluye que la supervivencia, rentabilidad y competitividad de la MiPyME dependerá en gran medida de una gestión estratégica eficiente, que promueva el fortalecimiento de sus capacidades nucleares, especialmente de aquellas que permitan potenciar la actividad innovadora; lo cual implica trabajar en la creación de una cultura y estructura organizacional que propicien la innovación e invertir en capital humano y en nuevas tecnologías que en su conjunto coadyuven a elevar la competitividad. De igual forma, construir el capital organizacional y relacional necesario para establecer nuevos negocios, consolidarse y alcanzar un mejor desempeño.

Por otro lado, resaltar que el enfoque hacia la innovación para la mejora de la eficiencia productiva y la efectividad organizacional característica de la MiPyME, no alcanza para satisfacer las exigencias y aprovechar las oportunidades de un mercado globalizado y altamente dinámico, que demanda la modernización tecnológica y la oferta constante de nuevos productos y servicios. De ahí, que a los directivos de las organizaciones correspondan los esfuerzos de liderazgo en la búsqueda de recursos estratégicos y establecimiento de acciones para elevar su tasa de innovación, especialmente de productos.

Asimismo, puntualizar que la edad y el tamaño de las organizaciones, son dos de las variables asociadas a la supervivencia y el éxito de una empresa, dado el conocimiento y aprendizaje adquirido a través de la exposición a los mercados; y las capacidades desarrolladas (de gestión, tecnológicas, mercadotecnia). En virtud de ello, puede afirmarse que sin importar el tamaño, las organizaciones deban trabajar en estrategias que lleven a su consolidación y crecimiento.

Por último, como limitaciones de este estudio, están el formular escalas más robustas para medir aspectos relacionados con el desempeño más allá de los aquí evaluados y los aspectos de tecnología, y cómo área de oportunidad, está el abordar el estudio de otros constructos como el liderazgo, la cultura organizacional y la gestión de conocimiento que pudiesen afectar directa o indirectamente estos resultados. 
FACTORES QUE INCIDEN EN EL DESEMPEÑO DE LA MIPYME DEL ESTADO DE QUERÉTARO.

\section{REFERENCIAS BIBLIOGRÁFICAS}

Aamir I., Satirenjit, K. y Mohammad, Y. (2017). Ownership Structure Attributes, Outside Board Members and SMEs Firm Performance with Mediating Effect of Innovation in Malaysia. Global Business and Management Research: An International Journal, 9(1), 393-402.

Aminreza, K., Maryam R., y Lagha A. (2011). Barriers to Innovatin Among Iranian SMEs. Asian Journal of Business Management, 3(2), 79-90. Recuperado de http://maxwellsci.com/print/ajbm/v3-79-90.pdf.

Amitabh, M. y Gupta, R. (2010). Research in strategy-structure-performance construct: Review of trends, paradigms and methodologies. Journal of Management and Organization, 16(5), 744-763.

Aral, S. y Weill, P. (2007). IT Assets, Organizational Capabilities, and Firm Performance: How Resource Allocations and Organizational Differences Explain Performance Variation. Organization Science, 18(5), 763-780.

Barney, J. (1991). Firm Resources and Sustained Competitive Advantage. Journal of Management, 17(1), 99-120.

Boyne, G., y Walker, M. (2004). Strategy Content and Public Service Organizations. Journal of Public Administration Research and Theory, 14(2), 231-252.

Brouthers, K., Nakos, G. y Dimitratos, P. (2015). SME Entrepreneurial Orientation, International Performance, and the Moderating Role of Strategic Alliances. Journal of Entrepreneurship Theory and Practice, 39(5), 1161-1187.

Bülbül H., Ömürbekm N., Paksoy T. y Bektas, T. (2013). An empirical investigation of advanced manufacturing technology investment patterns: Evidence from a developing country. Journal of Engineering and Technology Management, 30(2), 136-156.

Chan, J. y Seok, K. (2014). Structure and perceived performance in public organizations. Public Management Review, 16(5), 620-642.

Cokins, G. (2017). Strategic business management: From planning to performance. New York, NY: John Wiley \& Sons.

Damanpour, F. (2009). Combinative effects of innovation types and organizational performance: A longitudinal study of service organizations. Journal of Management Studies, 46(4), 650675.

Dyer, J., Gregersen, H., y Christensen, C. (2011). The Innovator's DNA - Mastering the Five Skills of Disruptive Innovators. Boston: Harvard Business School Publishing Corporation.

Edquist, C., (2001). Systems of innovation for development (SID). Background paper for chapter I: Competitiveness, innovation and learning: Analytical framework for the UNIDO World Industrial Development Report (WIDR).

Edvinsson, L. y Malone, M. (1999). El capital intelectual. Cómo identificar y calcular el valor de los recursos intangibles de su empresa. Barcelona, España: Ed. Gestión.

Estrada, B., García, D. y Sánchez, V. (2009). Factores determinantes del éxito competitivo de la Pyme: Estudio empírico en México. Revista Venezolana de Gerencia, 14(46), 169-182.

Gardiner, B. (2015). Workplace Culture in SMEs Thwarting Innovation. CIO from IDG. Recuperado de http://www.cio.com.au/article/570535/. 
FACTORES QUE INCIDEN EN EL DESEMPEÑO DE LA MIPYME DEL ESTADO DE QUERÉTARO.

Gica O. y Negrusa A. (2011). The Impact of Strategic Planning Activities on Transylvanian SMEsAn Empirical Research. Procedia Social and Behavioral Sciences, 24(1), 643-648.

Ghobakhloo, M., Hong, T., Sabouri, M, y Zulkifly, N. (2011). Information technology adoption in Small and Medium-sized Enterprises; An appraisal of two decades literature. Interdisciplinary Journal of Research in Business, 1(7), 53-80.

Glaister, K., Dincer, O., Tatoglu, E., Demirbag M. y Zaim, S. (2008). A causal analysis of form.al strategic planning and firm performance. Evidence from an emerging country. Management, 46(3), 365-391.

Grant, R. (1996). Toward a knowledge-based theory of firm. Strategic Management Journal, 17(52), 109-122.

Gronum, S., Verreyne, L., y Kastelle J. (2012). The role of networks in small and medium- sized enterprise and firm performance. Journal of Small Business Management, 50(2), 257-282.

Gruber, M. (2007). Uncovering the value of planning in new venture creation: A process and contingency perspective. Journal of Business Venturing, 22(6), 782-807.

Hair, J., Anderson R. E., Tatham R. L. y Black, C. (1999). Análisis Multivariante (5ª ed.). Madrid, España: Pearson Prentice Hall.

Heidenreich, M. (2009). Innovation patterns and location of European low- and mediumtechnology industries. Research Policy, 38(3), 483-494.

Hervas, J., Sempere, F. y Boranat, C. (2014). Process innovation strategy in SMEs, organizational innovation and performance: a misleading debate? Small Business Economics, 43(4), 873886.

Hoffman, W. y Schosser, R. (2001). Success Factors of Strategic Alliances in Small and Mediumsized Enterprises-An Empirical Survey. Long Range Planning, 34(3), 357-381.

Howard, E. (2014). Using Tech to Transform, Not Just Support, Your Core Processes. Credit Control, 35(1-2), 58-61.

Instituto Nacional de Estadística, Geografía e Informática (2019). Directorio Estadístico Nacional de Unidades Económicas, DENUE Interactivo, INEGI.

Instituto Nacional de Estadística, Geografía e Informática (2014). Micro, pequeña, mediana y gran empresa. Estratificación de los establecimientos. Censos Económicos. INEGI.

Jeffers, P., Muhanna, W., y Naul B. (2008). Information Technology and Process Performance: An Empirical Investigation of the Interaction Between IT and Non-IT Resources, Decision Science, 39(4), 703-735.

Jiménez, D. y Sanz, R. (2011). Innovation, organizational learning, and performance. Journal of Business Research, 64(4), 408-417.

Kanten, P., Kante, S., y Gurlekc, M. (2015). The Effects of Organizational Structures and Learning Organization on Job Embeddedness and Individual Adaptive Performance. Procedia Economics and Finance, 23(1), 1358-1366.

Kaplan, R. y Norton D. (2008). The execution premium, Boston, Massachusetts, USA: Harvard Business School Press.

Kennedy, G. (2006). Introduction of Advanced Manufacturing Technology: a literature review. Sabaragamuwa University Journal, 6(1), 116-134. 
FACTORES QUE INCIDEN EN EL DESEMPEÑO DE LA MIPYME DEL ESTADO DE QUERÉTARO.

Kotha, S. (1991). Strategy, manufacturing structure, and advanced manufacturing technologies. Best Paper Proceedings Academy of Management, 293-297.

Kraus, S., Harms, R. y Schwarz, E. (2006). Strategic planning in smaller enterprises-New empirical findings. Management Research News, 29, 334-344.

Laforet, S., y Tann, J. (2006). Innovative characteristics of small manufacturing firms. Journal of Small Business and Enterprise Development, 13(3), 363-380.

Lee, C. y Yang, H. (2011). Organization structure, competition and performance measurement systems and their joint effects on performance. Management Accounting Research, 22, 84-104.

Leyva, A., Cavazos, J. y Espejel, J. (2018). Influencia de la planeación estratégica y habilidades gerenciales cómo factores internos de la competitividad empresarial de las Pymes. Contaduría y Administración, 63(3), 1-21.

Liao, Ch., HuiChuang, S., y Laitoc P. (2011). How knowledge management mediates the relationship between environment and organizational structure. Journal of Business Research, 64(7), 728-736.

Mairesse, J., Mairesse, J. y Robin, S. (2009). Innovation and productivity: a firm-level analysis for French Manufacturing and Services using CIS3 and CIS4 data (1998-2000 and 20022004). Working paper. 1-15.

Martínez F., Sánchez A., Calvo S. y García D. (2007). Estrategia e innovación de la Pyme industrial en la comunidad de Cantabria: Un análisis comparado con España. Cátedra Pyme de la Universidad de Cantabria, Cantabria, España: Ediciones TGD.

Masocha, R. (2014). Interaction of Technological Marketing and Porter's Five Competitive Forces on SME Competitiveness in South Africa Progress Mediterranean. Journal of Social Sciences, 5(4), 254-262.

Mazzarol, T., Clark, D., Reboud, S., Gough, N., y Olson, P. (2014). Perceptions of innovation climate and the influence of others: A Multi-Country Study of SMEs. International Journal of Innovation Management 18(1), 1-24.

Minarelli F., Raggi M., y Viaggi, D. (2013). Network for innovation as a way to enhance competitiveness: an overview of Italian food SMEs Entering Networks. Second Conference Paper, Italian Association of Agricultural and Applied Economics, 149934, 1-10.

Mittal, S., Romero, D. y Wuest, T. (2018). Towards a Smart Manufacturing Toolkit for SMEs. Proceedings of the 15th International Conference on Product Lifecycle Management. Springer, 540(1), 476-487.

Mithas, S., Tafti, A., Bardhan, I., y Goh, M. (2012). Information technology and firm profitability: mechanisms and empirical evidence. MIS Quarterly 36(1), 205-224.

Ndemezo, E, y Kayitana, Ch. (2017). Innovation and Firms' Performance in the Rwandese Manufacturing Industry. A firm Level Empirical Analysis, 1-19. Recuperado de http://includeplatform.net/wp-content/uploads/2017/12/Research-Paper-on-Innovationand-firms-performance.pdf.

Nicolescu, O., y Nicolescu C. (2009). Procesual and Structural Organisational Features of Small Business. Review of International Comparative Management, 10(4), 585-597. 
FACTORES QUE INCIDEN EN EL DESEMPEÑO DE LA MIPYME DEL ESTADO DE QUERÉTARO.

OECD, 2009. Innovation firms: a microeconomic perspective. Paris, France: Organization for Economic Cooperation and Development. Recuperado de https://www.oecd.org/publications/innovation-in-firms-9789264056213.

Quinn, R. y Rohrbaugh, J. (1983). A spatial model of effectiveness criteria: Towards a competing values approach to organizational analysis. Management Science, 29(3), 363-377.

Sahay, Y. y Gupta, M. (2011). Role of organization structure in innovation in the bulk-drug industry. Indian Journal of Industrial Relations, 46(3), 450-464.

Savaneviciene, A, Rutelione, A., y Ciutiene, A. (2014). Crucial Transversal Competences in the Changing Environment: Case of the European SMEs Managers. Economics and Management, 19(1), 100-108.

Shapiro, D., Tang, Y., Wang, M., y Zhang, W. (2015). The effects of corporate governance and ownership on the innovation performance of Chinese SMEs. Journal of Chinese Economic and Business Studies, 13(4), 311-335.

Sharma, N. (2014). Determinants of innovation: A study of small and medium enterprises in India. World SME News (Publication of WASME), 5, 5-14.

Sosiawani, I., Ramli, A., Mustafa, M. y Yussoff, R. (2015). Strategic Planning and Firm Performance: A Proposed Framework. International Academic Research Journal of Business and Technology 1(2), 201-207.

Stock, M. y Zacharias, A. (2011). Patterns and performance outcomes of innovation orientation. Journal of the Academy of Marketing Science, 39(6), 870-888.

Street y Cameron. (2007). External relationships and the small business: A review of small business alliance and network research. Journal of Small Business Management, 45(2), 239-266.

Thi, T. y Nico, C. (2015). How Institutions Influence SME Innovation and Networking Practices: The Case of Vietnamese Agribusiness. Journal of Small Business Management, 53(1), 209-228.

Tran, Q. y Tian, Y. (2013). Organizational Structure: Influencing Factors and Impact on a Firm. American Journal of Industrial and Business Management, 3(2), 229-236.

Van de Vrande, V., De Jongb, J., Vanhaverbekec, W. y Rochmontd, M. (2009). Open innovation in SMEs: Trends, motives and management challenges. Technovation 29(1), 423-437. 